\title{
Interactive technologies to improve eating experiences in young professionals
}

\author{
Mario O. Parra, Luis A. Castro, Jesus Favela
}

Published: 30 November 2021

\begin{abstract}
In 2020, the COVID-19 pandemic has provoked changes in people's lifestyles around the world. This work presents results of a qualitative study about changes in young professionals' eating habits generated by the pandemic. Furthermore, this paper describes new scenarios for interactive technology designs to improve eating experiences. Lastly, future works on this topic is depicted.
\end{abstract}

\section{Keywords:}

Human-Computer Interaction; Eating experiences; Design opportunities.

\section{Introducción}

En el 2020, la pandemia por COVID-19 ha provocado cambios la vida cotidiana de las personas [2]. En un contexto local, México ha sido uno de los países más afectados por esta situación, ya que ha presentado más del doble de muertes en comparación con otros países de Latinoamérica [3].

El confinamiento en México fue menos estricto comparado con el resto de los países. Solamente los primeros 2 meses (después de haberse declarado pandemia mundial en marzo 2020) se tuvo restricciones fuertes [4]. Por ejemplo, las compañías privadas y no esenciales fueron obligadas a detener sus actividades por 70 días. Después de eso, el gobierno mexicano utilizó un sistema de semáforos para restablecer las actividades de forma escalonada en todo el país.

Antes de la pandemia por COVID-19, la población mexicana ya presentaba problemas de obesidad provocados por sus malos hábitos alimenticios y la falta de ejercicio físico. Sin embargo, con la situación actual del mundo, se espera una mayor preocupación de las personas por mejorar su salud y mantener un sistema inmunológico fuerte, esto con el propósito de disminuir la posibilidad de sufrir daños fuertes en el organismo en caso de ser infectado por el virus del COVID-19.

Parra, Mario O., Favela, Jesús

CICESE

Ensenada, México.

marioparra@cicese.edu.mx,favela@cicese.mx

Castro, Luis A.

Instituto Tecnológico de Sonora (ITSON)

Cd. Obregón, México

luis.castro@acm.org
Por estos motivos, la situación actual mundial y de México incitan a realizar investigaciones enfocadas en mejorar la experiencia de comer de los mexicanos. No obstante, la unión de la alimentación y la tecnología es una posición compleja que conlleva muchos retos distintos. Las actividades que existen alrededor de la comida son varias y cada una tiene sus desafíos individuales. Por ejemplo, el monitoreo de alimentación automático es el área que se encarga de investigar e identificar los momentos exactos en que una persona ingiere sus alimentos [5].

Durante la elaboración de este trabajo de investigación, se pretende diseñar y evaluar tecnologías interactivas con la capacidad de mejorar la experiencia alrededor de la alimentación de las personas en México. Para ello, se definen las siguientes preguntas de investigación:

- ¿Cuál es la situación actual de los mexicanos con respecto a sus hábitos alimentarios y el uso de tecnologías durante la pandemia?

- ¿Qué tecnologías interactivas ayudarían a mejorar la experiencia de comer de los mexicanos?

- ¿Cuáles son las soluciones tecnológicas que tienen la capacidad de persuadir a las personas a mantener una alimentación saludable en su vida diaria?

\section{Trabajo Relacionado}

En la última década, dentro del área de Interacción Humano Computadora se han creado varias soluciones de actividades relacionadas con la alimentación. Existe un término denominado HFI (Human-Food-Interaction) con el cual varios investigadores han logrado clasificar los trabajos recientes en HCI que se relacionan con la actividad de comer [6][7].

Los enfoques de los trabajos de alimentación pueden clasificarse en distintos conglomerados. Por ejemplo, existen trabajos enfocados en el monitoreo de alimentación con dispositivos vestibles [5], otros en aplicaciones que apoyan con la adherencia a nuevas dietas alimenticias [8], otros en el registro de alimentación [9], y otros en el aspecto social alrededor de los momentos de comida [10].

A partir del año 2020, por motivos de la pandemia por COVID19, están surgiendo nuevas investigaciones con el propósito principal de entender los cambios de hábitos de alimentación que pueden existir por el confinamiento (por ejemplo: [11]). Esto posteriormente, debe ser enlazado con posibles soluciones tecnológicas capaces de mejorar (o mantener) los hábitos de alimentación de las personas. 


\section{Metodología de la investigación}

La Figura 2 muestra el proceso que se planea seguir como metodología de la investigación. A continuación, se detalla cada punto en los párrafos posteriores.

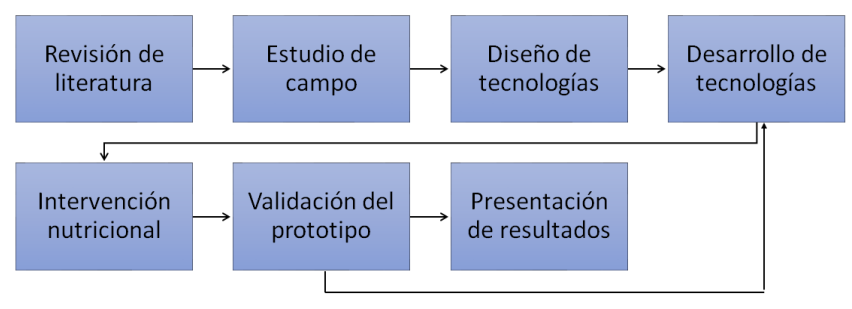

Figura 1. Metodología de la Investigación

- Revisión de la literatura. Recopilar información del estado del arte de artículos relevantes al uso y aplicación de tecnologías interactivas para mejorar la experiencia de comer de las personas.

- Estudio de campo. Realizar un estudio con personas en México para comprender sobre los procesos y hábitos alimentarios correspondientes a la situación actual. Para ello, es necesario aplicar entrevistas a los participantes con el propósito de recolectar datos cualitativos relacionados con los hábitos de alimentación actuales de las personas.

- Diseño de tecnologías. Diseñar tecnologías interactivas para mejorar la experiencia de comer en adultos jóvenes. Para realizar el diseño, es necesario recibir retroalimentación de expertos en la materia y posibles usuarios futuros de las aplicaciones.

- Desarrollo de tecnologías. El propósito principal será implementar las tecnologías obtenidas de análisis del estudio y las sesiones de diseño.

- Intervención nutricional. Reclutamiento de participantes para probar el uso de las tecnologías en un laboratorio o en un ambiente natural del individuo.

- Validación del prototipo. Adaptación de las tecnologías con los usuarios de acuerdo a la retroalimentación recibida durante la intervención. Se realizarán pruebas y evaluarán los resultados para encontrar un prototipo que arroje mejores resultados.

- Presentación de resultados. Presentar los resultados de la intervención con análisis de datos cuantitativos y cualitativos. Además, presentar el diseño y la estructura del prototipo final en revistas importantes de tecnología.

\section{Calendario de Actividades}

La Figura 2 presenta el calendario de actividades para los 4 años correspondientes al doctorado. Actualmente, ya se ha trabajado y se han presentado resultados para los 3 primeros puntos: revisión de literatura, estudio de campo y presentación de propuesta. En estos momentos, el trabajo reciente está siendo enfocado en el diseño de las tecnologías. Este último punto requiere de la planeación y colaboración en sesiones de diseño con expertos y posibles usuarios de las tecnologías.

\begin{tabular}{|l|c|c|c|c|c|c|c|c|c|c|c|c|c|}
\hline \multirow{2}{*}{ Actividades } & \multicolumn{2}{|c|}{ Primer Año } & \multicolumn{2}{|c|}{ Segundo año } & \multicolumn{2}{|c|}{ Tercer Año } & \multicolumn{2}{|c|}{ Cuarto Año } \\
\hline & 1 & 2 & 3 & 1 & 2 & 3 & 1 & 2 & 3 & 1 & 2 & 3 \\
\hline Revisión de literatura & & & & & & & & & & & & \\
\hline Estudio de campo & & & & & & & & & & & & \\
\hline Presentación de propuesta & & & & & & & & & & & & \\
\hline Diseño de tecnologías & & & & & & & & & & & & \\
\hline Desarrollo de tecnologías & & & & & & & & & & & & \\
\hline Validación del prototipo & & & & & & & & & & & & \\
\hline Intervención Nutricional & & & & & & & & & & & & \\
\hline Análisis y resultados & & & & & & & & & & & & \\
\hline Mejora de prototipo & & & & & & & & & & & & \\
\hline Escritura de artículos & & & & & & & & & & & & \\
\hline Escritura de tesis / Defensa & & & & & & & & & & & & \\
\hline
\end{tabular}

Figura 2. Calendario de Actividades

\section{Estudio de Campo}

Se realizó un estudio cualitativo para comprender el impacto de la pandemia por COVID-19 en el estilo de vida de las personas y en su alimentación. Para ello, se reclutó a 14 profesionistas jóvenes mexicanos (ver Tabla 1) con una edad promedio de 26 años y se les aplicó una entrevista semiestructurada con los siguientes temas: estilo de vida, hábitos alimenticios y uso de tecnologías.

Por motivos del confinamiento, las entrevistas fueron llevadas a cabo por videoconferencia y tuvieron una duración promedio de 36 minutos $( \pm \quad 11.5$ minutos $)$. Posteriormente, con las transcripciones se realizó un proceso de codificación línea por línea para obtener los distintos temas provenientes de las respuestas de los informantes. A continuación, se describen de forma resumida los 4 temas principales obtenidos de las entrevistas.

Tabla 1. Participantes del Estudio.

\begin{tabular}{|c|c|c|c|}
\hline Participante & Género & Edad & Profesión \\
\hline P1 & F & 27 & Maestra \\
\hline P2 & F & 25 & Ventas \\
\hline P3 & M & 28 & Estudiante Posgrado \\
\hline P4 & F & 27 & Diseñador Gráfico \\
\hline P5 & M & 25 & Estudiante Posgrado \\
\hline P6 & M & 31 & Reclutador \\
\hline P7 & M & 23 & Estudiante Posgrado \\
\hline P8 & M & 28 & Estudiante Posgrado \\
\hline P9 & M & 25 & Psicólogo \\
\hline P10 & M & 28 & Nutriólogo \\
\hline P11 & F & 25 & Abogada \\
\hline P12 & F & 26 & Maestra \\
\hline P13 & M & 25 & Ingeniero \\
\hline P14 & M & 30 & Trabajador de Hotel \\
\hline
\end{tabular}

\subsection{Sentimientos negativos afectan patrones de alimentación}

Para algunos de nuestros participantes la pandemia provocó sentimientos de ansiedad por estar en una situación desconocida, o sentimientos de preocupación por la salud de sus seres queridos. Este tipo de sentimientos negativos, ligado con el aumento de tiempo libre por motivos del confinamiento, puede afectar en otros comportamientos de la persona. Por ejemplo, un participante hombre de 31 años, mencionó lo siguiente:

"Lo que pensaba mi mente era, quédate acostado todo el día, estate durmiendo, ponte a ver series, todo el día desde que amanece hasta 
que te duermas, y estate levantando nada más a comer y a volverte acostar"

En esta oración es posible notar el estado mental negativo en el que se encontraba esta persona. Con relación a su contexto del momento, el participante trabaja de reclutador y sus actividades recién se modificaron a estar desde casa todo el tiempo. Esto pudo ser un disparador para mostrar cierta apatía, e incluso hasta indicios de depresión. Además, todo esto se liga a la forma de comer de la persona, como menciona que no es consciente en su forma de comer, simplemente lo hacía sin pensar.

\subsection{Encontrar motivación para mejorar su salud}

Otro tema de interés fue cómo varios participantes comenzaron a preocuparse más por mejorar su salud y buscar soluciones en su tiempo libre. Además, la situación del confinamiento provocó que la mayoría de las personas pasaran más tiempo en sus hogares, lo cual a su vez generó más situaciones de tiempo libre para algunos. Por ejemplo: un participante ingeniero de 23 años explica:

"Antes de la pandemia, yo hacía nulo ejercicio físico la verdad. Y durante la pandemia, tenía tanto tiempo libre que decidi ejercitarme en casa. Entonces, utilizaba una aplicación que me daba ejercicios para casa todos los días, y es lo que utilizaba con mi propio peso. Pero, pues ya llevo un mes que he visitado la nutrióloga y tengo un plan de entrenamiento y de nutrición en conjunto, que van enfocados a las mismas metas. “

Este participante aprovechó la situación actual para buscar ayuda profesional de un nutriólogo y buscó aplicaciones tecnológicas para mejorar su actividad física. Aquí, es importante resaltar el contraste con el participante del punto anterior (3.1), esto quiere decir, que la forma de actuar de cada persona puede tener un impacto positivo o negativo disparados por situaciones la misma situación de la pandemia.

\subsection{Aumento de cocinar y comer en casa}

El confinamiento del país forzó a la mayoría de los participantes a comer más en su casa y tener que cocinar sus propios alimentos. De hecho, se espera que este tipo de actividades de cocinar tenga un impacto positivo en las personas, ya que los problemas mencionados de obesidad en México, en muchas ocasiones son causados por el tipo de alimentos altos en calorías que se adquieren en restaurantes y puestos de comida. Como ejemplo, un participante hombre de 29 años mencionó lo siguiente:

"Curiosamente noté que podía consumir más alimentos cocinados por mí, yo creo que esa parte es interesante porque, si hacia comida [antes de la pandemia], pero pues una vez para toda la semana y mi variedad de alimentos era menor, y cuando cambio a la pandemia mi variedad de alimentos fue mayor"

Para este participante, su aumento de tiempo para cocinar sus alimentos también ayudó a aumentar su variedad en la alimentación. Sin embargo, otros participantes también mencionaron que podían cocinar más platillo, pero que solían elegir comidas que se caracterizan por altas en grasas (ejemplo: hamburguesas).

\subsection{Reducción de socializar al comer}

Otro cambio importante que generó la situación de la pandemia fue que disminuyeron oportunidades para socializar de las personas. Como explicaron algunos, la hora de la comida era un momento en el que solían socializar con sus amigos y seres queridos, y por la situación actual, ya no es posible hacerlo libremente. Por ejemplo, un estudiante de posgrado en computación de 28 años explicó su situación:
"Yo creo que el cambio mental ha sido de la visón que tienes respecto a las actividades sociales. Antes, era muy frecuente decir vamos a comer, vamos por una pizza, vamos por alguna cerveza, o algo por el estilo, y ahorita pues ya no".

En esta cita, es claro notar el cambio mental del participante. Él solía aprovechar el tiempo de comida para socializar con sus amistades, pero ya no lo podía hacer como antes. Este es solo un ejemplo de varias situaciones ejemplificadas en las experiencias de los participantes con respecto a la reducción de sus actividades sociales, muchas de ellas giraban alrededor de las horas de comida, y el comer era un simple pretexto para estar con sus amistades o seres queridos.

\section{Oportunidades de Diseño}

Los 4 temas principales obtenidos del estudio cualitativo ayudaron a identificar distintos escenarios que viven las personas actualmente. Con estos escenarios, se llevaron a cabo sesiones de ideas de diseño con personas con conocimiento tecnológico para obtener una solución tecnológica que ayude a mejorar la experiencia de comer de las personas. Primeramente, se realizaron lluvia de idea con el autor de este trabajo en conjunto con sus asesores. Posteriormente, se realizaron de forma virtual, 2 sesiones de ideas con personas con conocimientos en tecnologías. La primera sesión con 3 programadores y la segunda con 2 diseñadoras de aplicaciones. A continuación, se presentan los escenarios con una posible solución tecnológica.

- Escenario 1: Las roommates de Susy regresaron a sus ciudades por motivos de la pandemia, y ella se quedó sola en su departamento. Esto ha provocado que Susy se sienta muy sola, sobre todo al momento de comer, ya que solía estar en compañía de alguna de sus compañeras.

La solución tecnológica pensada para este escenario se tituló "MesaPara3". Esta aplicación permitiría a sus usuarios entrar a una sala de audio de forma remota y conectarse con otras 2 personas al momento de comer. De esta manera, cada usuario desde su hogar puede entablar conversaciones con conocidos o desconocidos a la hora de la comida, lo cual ayudaría a los participantes a sentirse menos solos.

- Escenario 2: A Miguel le suelen ocurrir episodios de comer compulsivamente, sobre todo cuando se distrae con tecnología y no nota las cantidades de comida que consume. Además, por motivos del confinamiento, Miguel pasa más tiempo en su casa y esto provoca que le ocurran más episodios.

La solución pensaba se titulo "JustEating". Este funciona como un módulo de un agente conversacional (ejemplo: Alexa) para guiar al usuario en los momentos de la comida. Este módulo se encargará de reproducir audios que ayuden a la persona a comer conscientemente y no distraerse con otras situaciones.

- Escenario 3: A la señora Dorita le gusta mucho cocinar para su familia, sin embargo, sus hijos y nietos ya no pueden visitarla seguido por motivos de la pandemia. Por lo tanto, en la mayoría de las ocasiones no tiene con quien compartir su comida, y ella está acostumbrada a cocinar para varias personas y ver sus expresiones de gusto.

Aquí se pensó una app que se llamaría “¡Ven por tu comida!”. En esta aplicación, a las personas que les gusta cocinar y les suele sobrar comida, les permitiría conectarse con otros usuarios consumidores de comida casera. Se espera que los usuarios consumidores sean personas adultas jóvenes que extrañen el sabor de la comida casera de sus hogares. 
- Escenario 4: Joaquín ha extrañado salir todos los fines de semana y poder socializar libremente con otras personas. Por motivos del confinamiento, las salidas de Joaquín han sido limitadas. Aunque lo que más extraña hacer de sus salidas, es probar los distintos restaurantes que hay en su ciudad y comparar opiniones con sus amigos.

La solución innovadora para este escenario se titula "Restaurante EnLínea". Esta solución aprovecha los entornos interactivos que para socializar de forma virtual distinta a las aplicaciones convenciales como Zoom o Meet (ejemplo: gather.town). Trata de un entorno virtual parecido a un videojuego, en el que cada persona tiene un avatar que se mueve con el mouse o teclado. Este avatar al estar cerca de otras personas tiene acceso a su cámara y micrófono para interactuar con ellos. De esta forma, la presente idea ayudaría a hacer la experiencia de un restaurante virtual más llamativa. Se necesitaría diseñar el espacio con mesas y sillas de un restaurante, entrar a ese mundo virtual con tu avatar, escoger una mesa con tus amigos, pedir tu comida en el mismo entorno, pero lo ideal sería que la comida le llegue a cada persona hasta su casa (si es posible).

\section{Trabajo Futuro}

Como trabajo futuro se espera la validación e implementación de las ideas tecnológicas generadas del estudio. Para ello, se requiere realizar talleres con posibles usuarios de los sistemas en conjunto con personas con conocimientos en creación de prototipos.

Posteriormente, las ideas generadas deberán ser descritas formalmente, con las funcionalidades correspondientes, y diseñar prototipos de baja calidad para corroborar la intención de uso y función en posibles usuarios de los sistemas.

Además, al implementar prototipos con mejoras $\mathrm{y}$ adaptaciones, será necesario evaluar con participantes la inclusión de este tipo de tecnologías en la vida diaria de las personas. Estas tecnologías deberán tener un impacto positivo con relación a la mejora de la experiencia de la comida de las personas.

\section{Motivación para el Consorcio}

Para la fase en que se encuentra actualmente este trabajo, sería de mucha ayuda poder recibir consejos y retroalimentación de personas con experiencia, y expertas en el tema del diseño de tecnologías interactivas para un bienestar de las personas.

El poder ayudar con ejemplos de cómo guiar este trabajo para pulir de mejor manera las ideas, con procesos que vayan desde el surgimiento de la idea hasta la inclusión de este tipo de nuevas tecnologías en las vidas de las personas.

Además, es indispensable poder acotar este trabajo desde fases tempranas. Actualmente el tema de alimentación es un área muy amplia que puede abarcar muchos retos individuales y distintos en cada momento. Por ejemplo, para algunas personas el hacer uso de tecnologías al momento de comer les parece una distracción y no le encuentran un beneficio, sin embargo, el propósito de este trabajo es encontrar la forma de beneficiar la vida de las personas con la mejora en su alimentación.

\section{Referencias}

[1] J. Dávila-Torres, J. De Jesús González-Izquierdo, and A. Barrera-Cruz, "Medicina social Panorama de la obesidad en México,” Apr. 2015.

[2] M. Ciotti, M. Ciccozzi, A. Terrinoni, W.-C. Jiang, C.-B. Wang, and S. Bernardini, "The COVID-19 pandemic," Crit. Rev. Clin. Lab. Sci., vol. 57, no. 6, pp. 365-388, Aug. 2020, doi: 10.1080/10408363.2020.1783198.

[3] A. Pablos-Méndez, J. Vega, F. P. Aranguren, H. Tabish, and M. C. Raviglione, "Covid-19 in Latin America," The BMJ, vol. 370. BMJ Publishing Group, Jul. 27, 2020, doi: 10.1136/bmj.m2939.

[4] I. Ibarra-Nava, J. A. Cardenas-De La Garza, R. E. RuizLozano, and R. G. Salazar-Montalvo, "Mexico and the COVID-19 Response," Disaster Med. Public Health Prep., vol. 14, no. 4, pp. E17-E18, Aug. 2020, doi: 10.1017/dmp.2020.260.

[5] [T. Vu, F. Lin, N. Alshurafa, and W. Xu, "Wearable Food Intake Monitoring Technologies: A Comprehensive Review," Computers, vol. 6, no. 1, p. 4, Jan. 2017, doi: 10.3390/computers6010004.

[6] [R. A. Khot and F. Mueller, "Human-food interaction," Found. Trends Human-Computer Interact., vol. 12, no. 4, pp. 238-415, 2019, doi: 10.1561/1100000074.

[7] [F. A. Bertran, S. Jhaveri, R. Lutz, K. Isbister, and D. Wilde, "Making sense of human-food interaction," in Conference on Human Factors in Computing Systems - Proceedings, May 2019, pp. 1-13, doi: 10.1145/3290605.3300908.

[8] [Q. Wang, B. Egelandsdal, G. V. Amdam, V. L. Almli, and M. Oostindjer, "Diet and physical activity apps: Perceived effectiveness by app users," JMIR mHealth uHealth, vol. 4, no. 2, p. e5114, Jun. 2016, doi: 10.2196/mhealth.5114.

[9] [P. Achananuparp, E. P. Lim, and V. Abhishek, "Does journaling encourage healthier choices? Analyzing healthy eating behaviors of food journalers," in ACM International Conference Proceeding Series, Apr. 2018, vol. 2018-April, pp. 35-44, doi: 10.1145/3194658.3194663.

[10] [C. Spence, M. Mancini, and G. Huisman, "Digital Commensality: Eating and Drinking in the Company of Technology," Frontiers in Psychology, vol. 10. Frontiers Media S.A., p. 2252, Oct. 09, 2019, doi: 10.3389/fpsyg.2019.02252.

[11] [L. Di Renzo et al., "Eating habits and lifestyle changes during COVID-19 lockdown: An Italian survey," J. Transl. Med., vol. 18, no. 1, p. 229, Jun. 2020, doi: 10.1186/s12967020-02399-5.

[12] [V. Flaudias et al., "COVID-19 pandemic lockdown and problematic eating behaviors in a student population," $J$. Behav. Addict., vol. 9, no. 3, pp. 826-835, Sep. 2020, doi: $10.1556 / 2006.2020 .00053$.

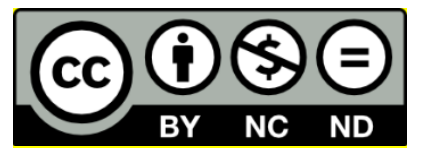

(C) 2021 by the authors. This work is licensed under the Creative Commons AttributionNonCommercial-NoDerivatives 4.0 International License. To view a copy of this license, visit http://creativecommons.org/licenses/by-nc-nd/4.0/ or send a letter to Creative Commons, PO Box 1866, Mountain View, CA 94042, USA. 